\title{
Caractérisation des champs de pression moyens et fluctuants à la surface des grands aéroréfrigérants
}

\section{Characterization of average and fluctuating pressure fields on the surface of large air-air heat-exchangers}

\author{
Jean-François Sageau \\ EDF - Direction des Etudes et Recherches \\ Service RNE \\ 6 , quai Watier - 78400 Chatou
}

\section{Introduction}

Il existe actuellement, dans la bibliographie, une disproportion considérable entre les efforts entrepris pour le calcul de la tenue au vent de la coque des réfrigérants et ceux destinés à étudier les forces qui s'exercent sur cette coque.

Cette disproportion est due principalement au fait que les champs de pression à la surface de la coque sont considérés comme connus depuis de nombreuses années.

Pourtant, les valeurs admises traditionnellement sont issues soit d'essais sur site sur des coques de petites tailles, soit d'essais en soufflerie utilisant des méthodes de simulation non validées.

L'augmentation de taille des coques de réfrigérants ces dernières années, qui s'est traduite par une augmentation des forces statiques et dynamiques exercées (fig. 1) sur la coque et par une modification de la géométrie de la structure ont conduit Electricité de France à remettre en cause les résultats obtenus antérieurement sur des coques de petites tailles.

Une étude à long terme, basée sur une double série d'essais sur site et en soufflerie a donc débuté en 1975, pour déterminer les champs de pression exercés par le vent sur la coque des grands réfrigérants.

Cette étude a d'ores et déjà fourni un certain nombre de résultats dont les principaux sont résumés dans ce texte.

\section{Position du problème}

L'écoulement, autour de la coque d'un réfrigérant présente 3 caractères spécifiques :

- Le nombre de Reynolds construit sur le diamètre moyen de la structure $(80<\bar{D}<150 \mathrm{~m})$ est de l'ordre de $10^{8}$.
Son ordre de grandeur, 2 à 3 fois supérieur à celui qu'il est possible de simuler dans les souffleries classiques, nécessite la mise en place de techniques de simulation lors d'essais en laboratoire.

Ces techniques doivent auparavant être validées par des essais sur site.

Par ailleurs, l'importance de ce nombre de Reynolds rend actuellement difficile une approche numérique du problème.

- L'écoulement autour de la coque est fondamentalement tridimensionnel.

Cette tridimensionnalité, qui est déjà caractéristique de l'écoulement amont (la couche limite atmo-

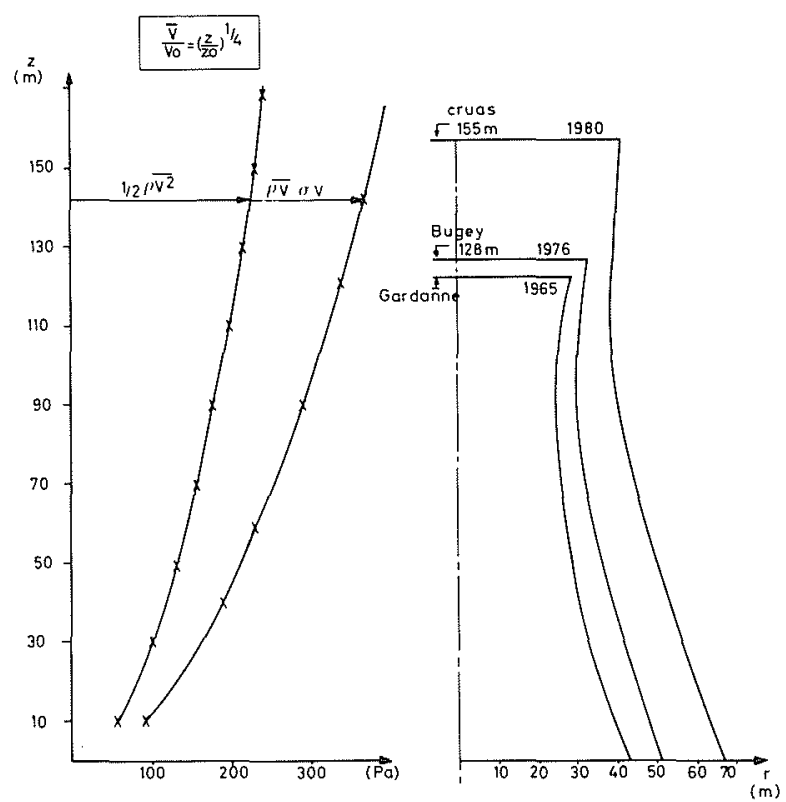

Figure 1 - Augmentation de la taille des aéroréfrigérants et des efforts statiques et dynamiques auxquels ils sont soumis 
sphérique) est encore accentuée par la géométrie de la coque et par son faible allongement.

Il est en conséquence difficile d'appliquer à l'écoulement une modélisation simple et le choix des grandeurs de référence utilisées pour normer les paramètres mesurés n'est toujours pas résolu.

- L'écoulement est instationnaire et les paramètres mesurés doivent être interprétés comme des variables aléatoires instationnaires.

En outre, l'intéraction entre le vent et la coque est caractérisée par le fait que les dimensions de la structure et celles des gros tourbillons de la turbulence atmosphérique sont du même ordre.

On retrouve donc, dans le champ de pression fluctuant à la surface de la coque, une influence prépondérante de la turbulence atmosphérique amont, et à un degré moindre, celle de l'échappement tourbillonnaire, qui est contrarié dans ce cas par la tridimensionnalité de l'écoulement.

La présence simultanée de ces trois caractères explique la complexité de l'écoulement autour des coques de réfrigérants et l'intérêt croissant qu'a suscité son étude depuis une quinzaine d'années.

\section{Méthode expérimentale utilisée}

La détermination des champs de pression exercés par le vent à la surface de la coque utilise deux moyens d'essais complémentaires :

- D'une part des mesures sur site, consistant à déterminer les champs de pression moyens et fluctuants à la surface de réfrigérants de type divers (lisses ou rugueux) placés dans des configurations variées (isolés ou en intéraction) -

Ces essais, lourds à mettre en œuvre, coûteux et d'une durée moyenne de 3 à 4 ans fournissent des valeurs de référence.

Ils sont utilisés à la fois pour déterminer les valeurs réelles des pressions et pour valider les techniques de simulation mises au point en laboratoire.

Le tableau 1 résume les études effectuées à ce jour par EDF. Elles concernent les réfrigérants de la centrale

Tableau 1 - Etudes effectuées en soufflerie (situation au 01/09/78)

\begin{tabular}{|c|c|c|c|c|}
\hline LSEU & CONF:GURATION & \begin{tabular}{|c|c|c|c|} 
DES \\
DE \\
SURACACE
\end{tabular} & $\begin{array}{l}\text { HAUTEGR } \\
(\mathrm{m})\end{array}$ & PARAMETRES MESURES \\
\hline GARDANNE & $1501 \mathbf{c}^{6}$ & Lisse & 120 & $\begin{array}{l}\text {-Ptession Externe- } \\
46 \text { capteurs } \quad \begin{array}{l}\text { i couronne } \\
3 \text { gentralfices }\end{array} \\
\text { Vent : } 5 \text { niveaux sur mât météorologique }\end{array}$ \\
\hline BUGEY & en intercelion & $\begin{array}{l}\text { Rugueux } \\
\text { (nervures) }\end{array}$ & 128 & 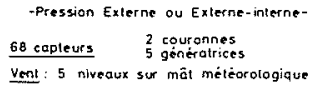 \\
\hline
\end{tabular}

ETUDES EFFECYUEES SUR SITE (STUATION AU O1/09/78)

\begin{tabular}{|c|c|c|c|c|}
\hline $\begin{array}{c}\text { LIEU } \\
\text { SIMULE }\end{array}$ & CONFIGUAATIOH & $\begin{array}{l}\text { HAUTEUR } \\
(\mathrm{m}) \\
(\mathrm{m})\end{array}$ & $\begin{array}{l}\text { PAAAAMETFE } \\
\text { SMMULES }\end{array}$ & PARAME TRES MESURES \\
\hline GARLANNE & Isolé & 122 & $\begin{array}{l}\text { Couche } \\
\text { camosphiori- } \\
\text { auve } \\
\text { Reynolds } \\
\text { per rugosi. } \\
\text { it }\end{array}$ & - Pression moyenne ou tluctuonie- \\
\hline CRUAS & En interaction & 155 & 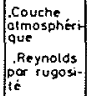 & $\begin{array}{c}\text {-Pression mayenne (5 niveaux de } 36 \text { copteurs) } \\
\text { ou } \\
\text { fluctuante (1 couronne de } 12 \text { caplours) }\end{array}$ \\
\hline
\end{tabular}

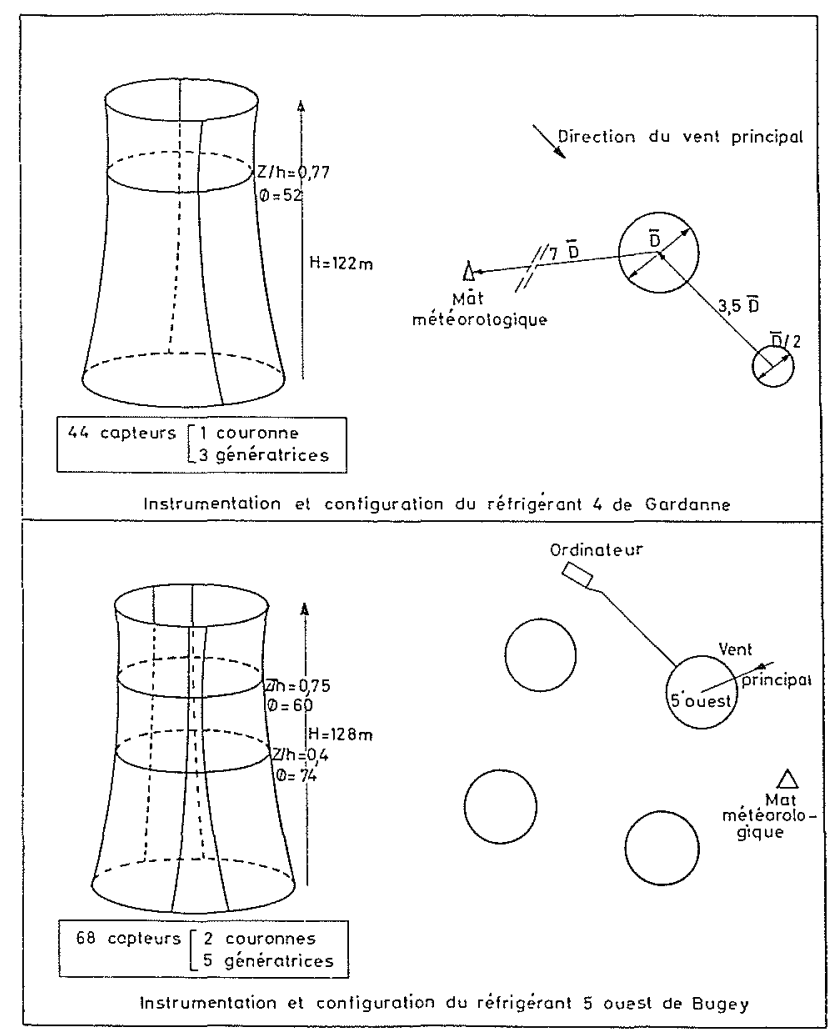

Figure 2

des Houillères de Provence à Gardanne et de la centrale nucléaire du Bugey (fig. 2).

Ces mesures nécessitent une instrumentation importante comportant un mât météorologique à l'amont et des capteurs de pression différentiels installés sur la coque.

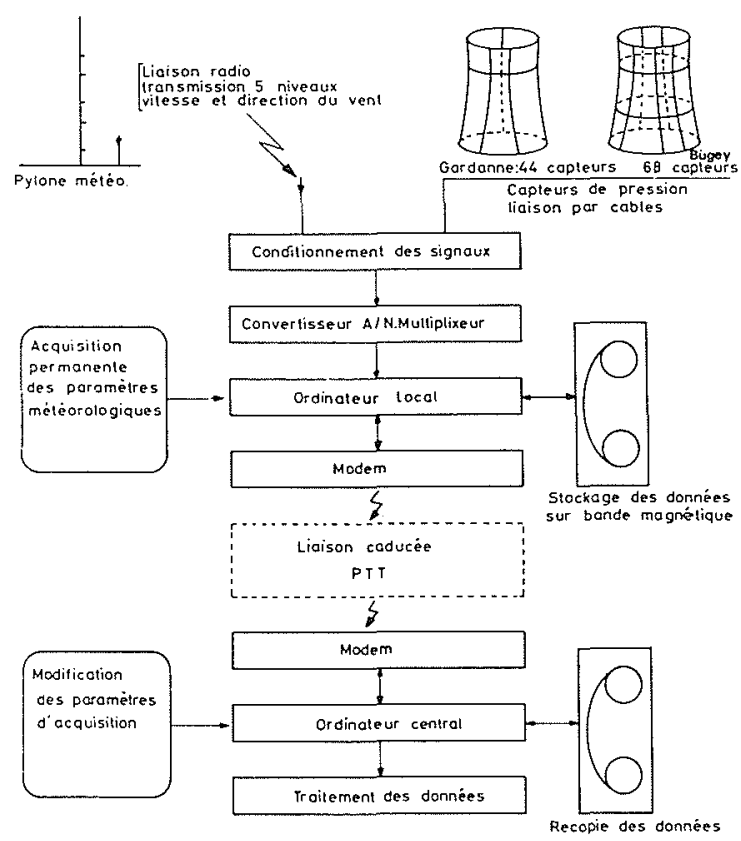

Figure 3 - Synoptique de la chaîne d'acquisition des mesures sur site 
L'instrumentation mise en place, gérée à partir du Laboratoire de Chatou par une liaison télé-informatique, (fig. 3), permet de mesurer les champs de pression externes (Gardanne) et externes ou internes (Bugey) sur 1 couronne (Gardanne) ou 2 (Bugey) et sur 3 (Gardanne) ou 5 (Bugey) génératrices, simultanément avec les caractéristiques du vent amont.

- D'autre part des mesures en soufflerie, menées parallèlement aux essais sur site.

Ces essais, d'une utilisation beaucoup plus souple sont utilisés pour mettre au point des techniques de simulation et pour fournir des données utiles pour l'interprétation des mesures in situ. A long terme, ces essais devront permettre de s'affranchir des essais "in situ".

Les mesures en soufflerie ont été effectuées à Modane, à l'institut Von Karman et à l'Ecole Centrale de Lyon. Ces diverses souffleries offrent des possibilités différentes : haut nombre de Reynolds, sans turbulence, à Modane ; nombre de Reynolds élevé avec turbulence à Von Karman ; nombre de Reynolds moyen avec couche limite atmosphérique à l'E.C.L. -

\section{Caractéristiques du champ de pression moyen.}

En chaque point de la coque, repéré par ses coordonnées $(\theta, z)$ le vent exerce une pression $p(\theta, z, t)$ qui, référencée à la pression atmosphérique amont $\overline{p o}(z)$, peut être décomposée en une composante moyenne $\bar{p}(\theta, z)$ et une composante fluctuante $p^{\prime}(\theta, z, t)$ :

$$
p(\theta, z, t)=\vec{p}(\theta, z)+p^{\prime}(\theta, z, t)
$$

Le champ de pression moyen $\vec{p}(\theta, z)$ exercé par le vent à la surface de la coque crée à l'intérieur de celle-ci un champ de contraintes statiques. Ce champ détermine à lui seul, actuellement, le dimensionnement des coques de réfrigérants.

Le champ de pression moyen $\bar{p}(\theta, z)$ agissant sur la coque est essentiellement influencé par cinq paramètres :

- le gradient de vitesse moyenne amont $\bar{V}(z)$,

- le nombre de Reynolds et l'état de surface,

- la géométrie de la coque,

- la configuration dans laquelle se trouve le réfrigérant.

Parmi ces cinq paramètres, il convient de faire jouer un rôle particulier au dernier. Dans le cas où un réfrigérant se trouve en configuration d'intéraction, il se trouve en effet soumis à un écoulement cisaillé très pertubé qui n'a plus de rapport avec la couche atmosphérique.

\section{Cas du réfrigérant isolé}

Dans le cas où le réfrigérant est isolé, on peut limiter a 3 les facteurs pouvant influencer le champ de pression dans la réalité :

le gradient de vitesse amont, l'état de surface et la géométrie de la coque.

Le nombre de Reynolds, qui reste toujours supérieur à $5.10^{7}$ pour les vitesses de vent intéressantes ne change pas d'ordre de grandeur d'un réfrigérant à l'autre.

En dehors des parties hautes et basses où l'écoulement est totalement tridimensionnel, on peut distinguer 3 régions autour de la coque (fig. 4 ).

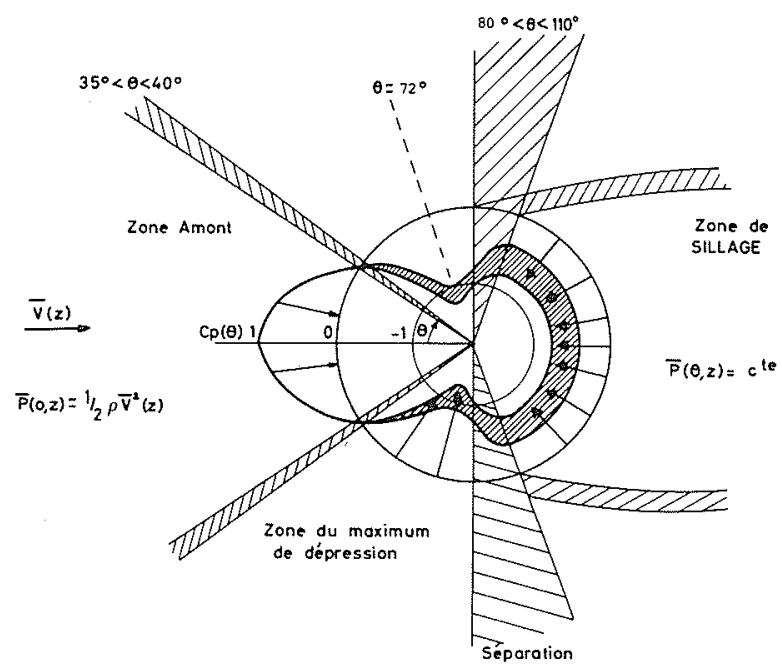

Figure 4 - Découpage en trois zones du champ de pression

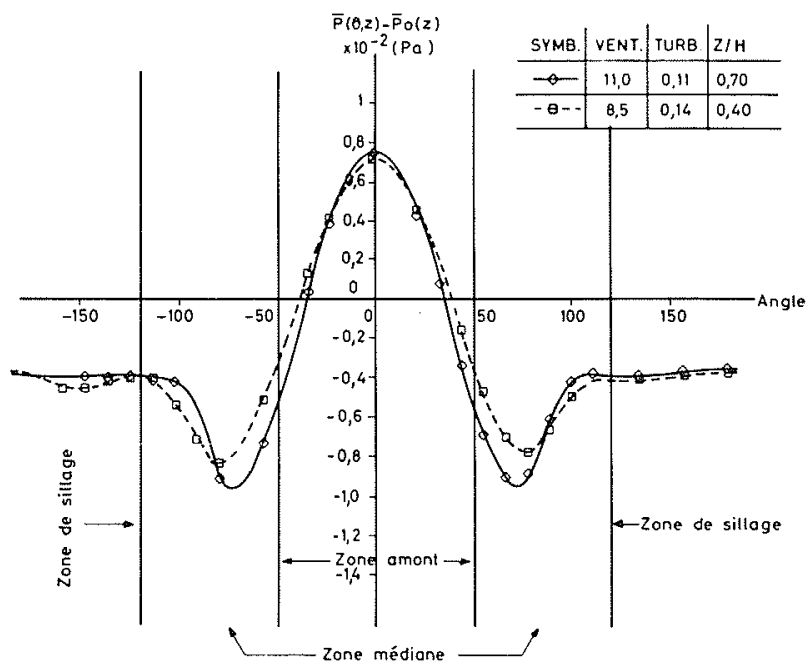

Figure 5 - Evolution de la pression moyenne sur 2 couronnes situées à $0,4 \mathrm{H}$ et $0,7 \mathrm{H}$

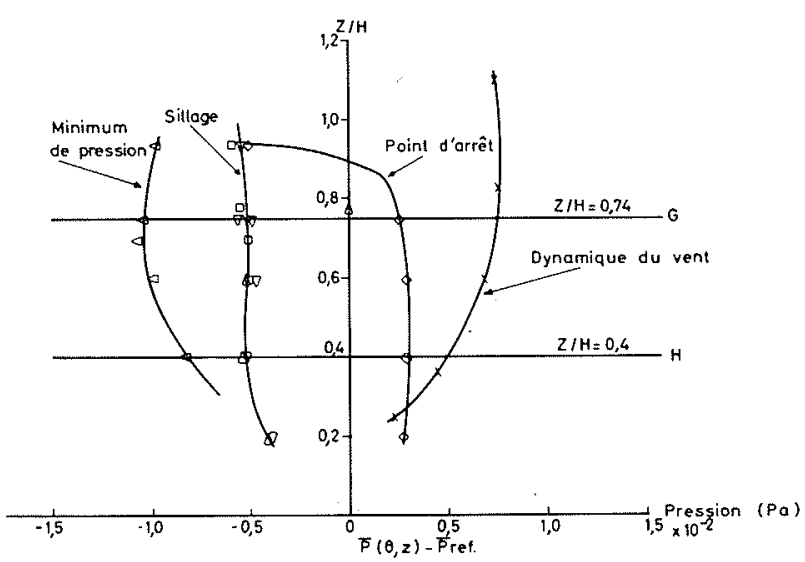

Figure 6 - Evolution de la pression en hauteur

- Dans la partie amont (Zone A), la pression moyenne est gouvernée par la dynamique du vent amont (à laquelle elle serait liée par une loi linéaire en l'absence de courbure de la coque [Réf. 1]) et par la courbure qui a tendance à uniformiser en hauteur la pression. 
Fig. $5 / 6$, on peut cependant trouver une relation entre la dynamique de l'écoulement amont à la même altitude et la pression d'arrêt :

$$
\bar{p}(\theta, z)=A(z) \times 1 / 2 \rho \bar{V}^{2}(z)
$$

la fonction $A(z)$ étant caractéristique de la géométrie de la coque.

Autour de ce point, la pression moyenne décroit avec l'azimut, avec un gradient de pression qui aug. mente en hauteur (fig. 5).

Dans toute cette zone, il existe une faible dispersion des mesures et on ne trouve pas d'influence de l'état de surface.

En haut de la tour, l'écoulement décolle et la pression moyenne chute brusquement.

- Dans la zone médiane (Zone B), située entre 50 et 110 degrés, se trouvent le minimum de pression et la zone de séparation.

Cette zone, où l'écoulement est fortement tridimensionnel, est caractérisée par une assez grande dispersion des valeurs mesurées.

La position du minimum de pression (fig. 5) se déplace vers l'avant de la coque, avec l'altitude, pour passer de 80 degrés environ à $0,4 \mathrm{H}$ à 72 degrés à $0,8 \mathrm{H}$ (H étant la hauteur de la tour).

$\mathrm{La}$ valeur de ce minimum augmente en valeur absolue en hauteur (jusqu'au col) mais il n'existe pas de relation directe entre cette valeur et la dynamique du vent amont à la même altitude.

Une première comparaison des résultats trouvés à Gardanne (réfrigérant lisse) et Bugey (réfrigérant rugueux) semble indiquer que la rugosité de surface n'a pas la même importance que celle trouvée en soufflerie (fig. 7).

La zone de séparation, caractérisée par un gradient de pression positif, garde une position fixée en hauteur.

- La zone arrière (Zone $\mathrm{C}$ ), qui correspond au sillage débute à environ 110 degrés du point d'arrêt. Elle est caractérisée par une pression constante sur environ les deux tiers de la tour á partir du sommet et quatre vingt degrés en azimut de part et d'autre du point de culot.

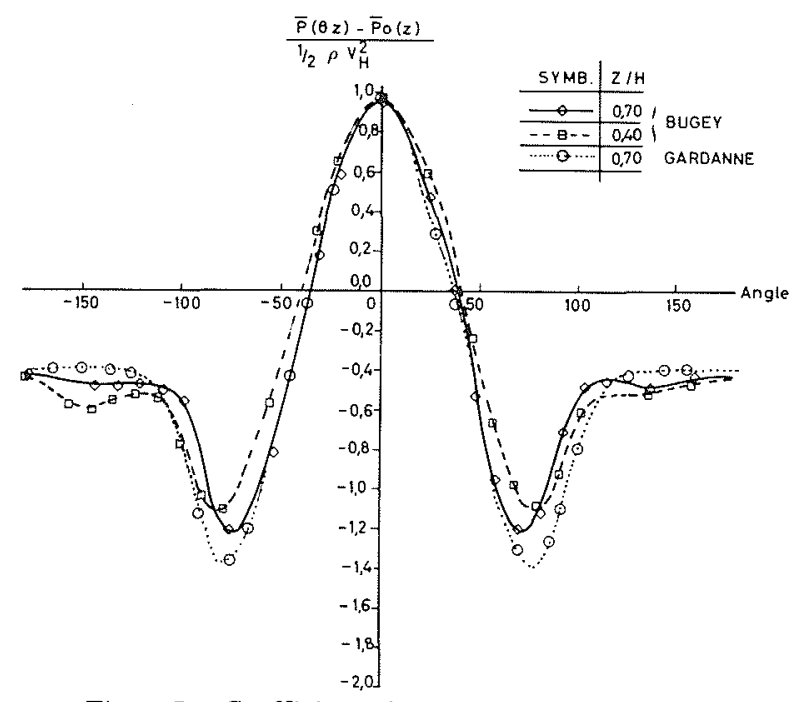

Figure 7 - Coefficients de pression obtenus sur site
Il semble que cette pression soit imposée à la fois par le décollement qui se produit en partie haute et par la géométrie de la tour, en particulier son allongement.

\section{Cas d'un réfrigérant en intéraction}

Dans le cas où plusieurs réfrigérants sont groupés sur le même site, il existe des directions de vent pour lesquelles certains réfrigérants se trouvent dans le sillage de tours plus ou moins proches.

Ces réfrigérants sont alors soumis à des écoulements très perturbés et le champ de pression autour de leur coque n'est plus gouverné par leur géométrie ou l'état de surface mais plutôt par la position relative qu'ils occupent par rapport à l'obstacle amont.

On peut distinguer, sur site comme en soufflerie, deux types d'interaction:

- une interaction forte, lorsque la distance de deux réfrigérants est suffisamment faible (inférieure à $2,5 \bar{D}$ ) pour que le seul paramètre significatif devienne l'angle d'intéraction (angle du vent et de l'axe des tours);

- une interaction faible, lorsque la distance des tours est suffisamment grande pour que la structure du champ de pression sur la tour aval ne dépende plus de l'angle d'interaction.

En cas d'interaction forte, le blocage de l'écoulement dû à la tour amont augmente jusqu'à la situation où la tour aval est entièrement dans le sillage de la première.

Sur le champ de pression moyen, cette évolution se traduit par des courbes de plus en plus dissymétriques et on observe (fig. 8) la disparition de l'un des pics de dépression, une forte déviation du point d'arrêt et un affaiblissement global de l'amplitude des pressions.

En cas d'interaction faible, les distributions azimutales de pression sont dans l'ensemble plus symétriques mais, du fait de la présence d'une zone de sillage à l'amont, l'amplitude des pressions dans la zone amont reste plus faible que celle constatée sur une tour isolée.

\section{Caractéristiques du champ de pression fluctuant}

Comme tout obstacle de section circulaire, d'allongement fini, plongé dans une couche turbulente, le

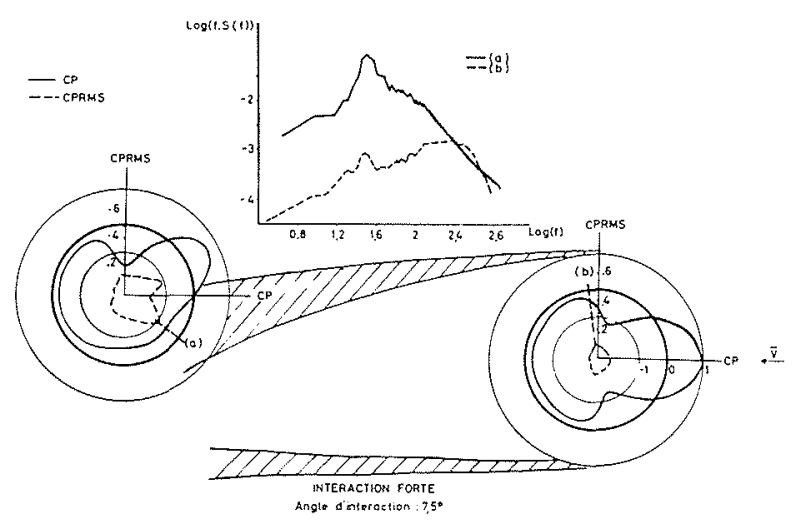

Figure 8 
champ de pression fluctuant autour de la coque d'un réfrigérant $\mathrm{a}$, en négligeant les fluctuations de pression pouvant provenir du mouvement de la coque, trois origines :

- la turbulence de l'écoulement amont

- la présence d'un détachement tourbillonnaire à l'arrière de la tour

- la présence d'un décollement en haut de la tour.

En configuration d'interaction, il faut ajouter à cellesci la présence, dans l'écoulement amont, de fluctuations de haut niveau.

L'originalité du problème posé par les coques de réfrigérants provient des dimensions de celles-ci ; dimensions qui, contrairement a celles d'obstacles de type cheminées, sont du même ordre de grandeur que celles des gros tourbillons de la turbulence amont.

Les différents phénomènes rencontrés sont donc intimement liés, mais on peut, par analogie avec les pressions moyennes, décrire le champ de pression fluctuant par ses caractéristiques dans les trois zones analysées précédemment.

\section{Fluctuations de pression dans la zone amont}

Dans la zone amont, la seule source de fluctuations est la turbulence atmosphérique amont. On retrouve donc, dans toute cette zone, des fluctuations de pression qui ont des caractéristiques semblables à celles de la turbulence amont.

En particulier, les densités de probabilité de la pression sont gaussiennes et les écarts-types, dont les ordres de grandeur sont fixés par celui de la turbulence amont, sont constants sur environ 40 degrés autour du point d'arrêt (fig. 9).

En comparant les spectres de pression ou les fonctions de répartition (fig. 10) obtenus dans cette zone avec ceux de la turbulence amont, à la même altitude, on observe que l'écoulement perd en hautes fréquences

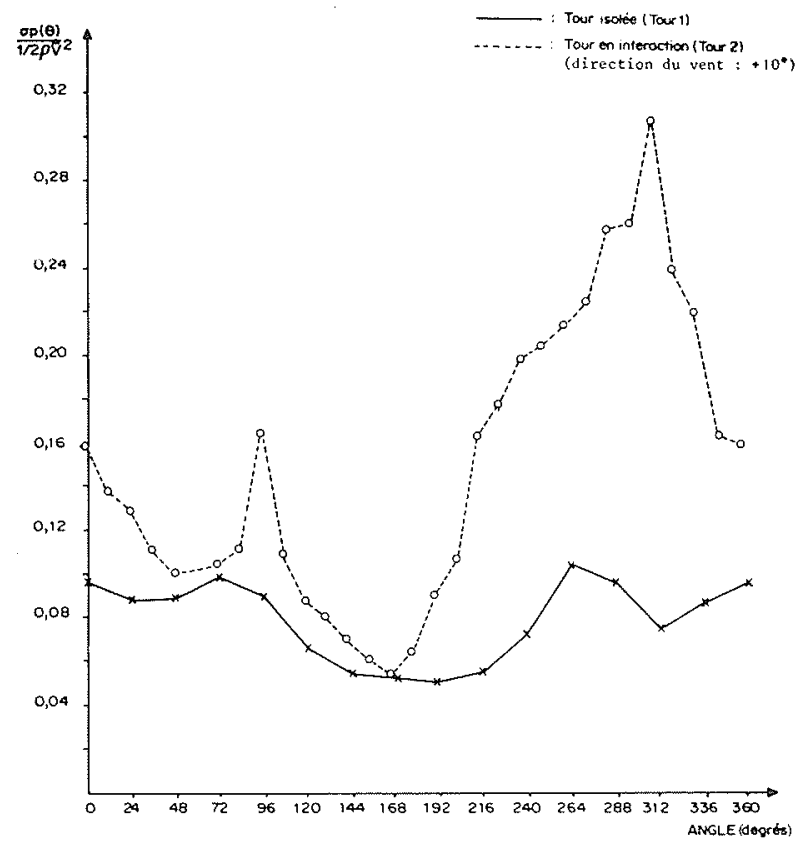

Figure 9 - Distribution des écarts-types de pression au col

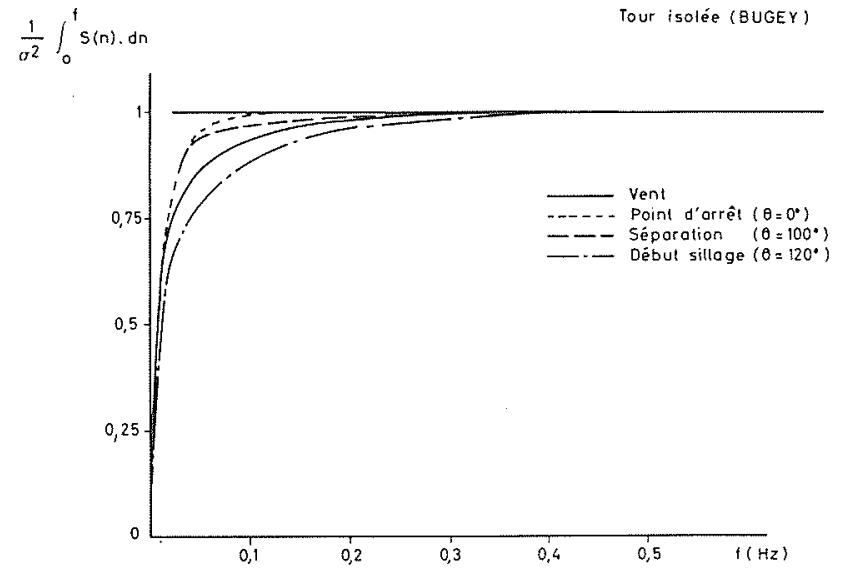

Figure 10 - Evolution des fonctions de répartitions spectrales en fonction de l'azimut

quand il approche de l'obstacle, tout au moins dans la zone du point d'arrêt.

Dans cette zone, les fluctuations de pression sont relativement bien corrélées en altitude, en dehors des parties hautes et basses de la tour, et les fluctuations sont en phase en hauteur.

En azimut, les corrélations sont données par la distribution, sur une couronne des valeurs :

$$
\rho\left(\theta_{o}, \theta, z\right)=\frac{\overline{p^{\prime}\left(\theta_{o}, z, t\right) p^{\prime}(\theta, z, t)}}{\sqrt{p^{\prime 2}\left(\theta_{o}\right)} \sqrt{p^{\prime}(\theta)}}
$$

$\theta_{0}$ étant l'azimut d'un point situé dans la zone du point d'arrêt.

On observe (fig. 11) que cette distribution est semblable à celle trouvée pour les pressions moyennes $\bar{p}(\theta, z)$. Cette similitude pourrait s'expliquer par le fait que le champ de pression moyen à la surface est gouverné par les grandes échelles de turbulence, échelles qui fixent les valeurs des corrélations spatiales.

\section{Fluctuations de pression dans la zone médiane (Zone B)}

Dans cette zone, et en particulier au niveau de la séparation, les fluctuations de pression augmentent brusquement (fig. 9).

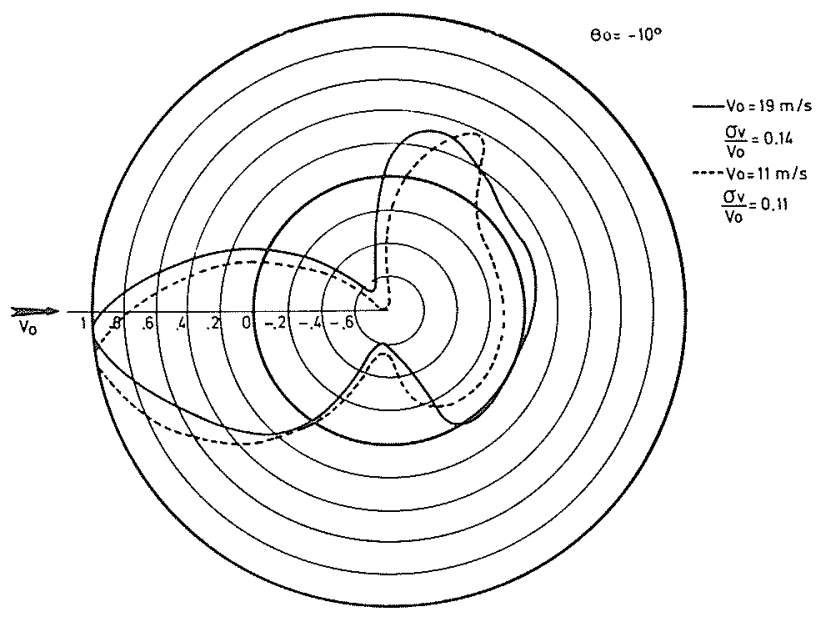

Figure 11 - Corrélation spatiale 


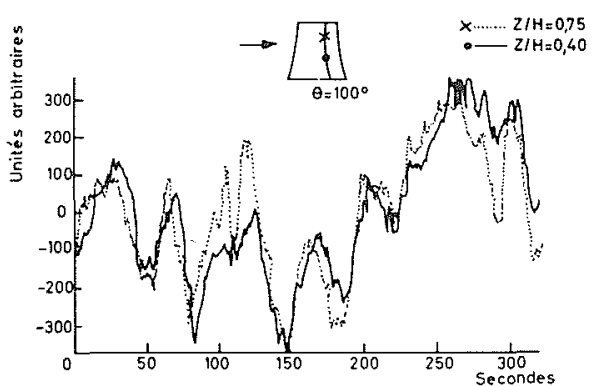

Comparaison des fluctuations de pression dons une zone de séparation a deux nauteurs distinctes

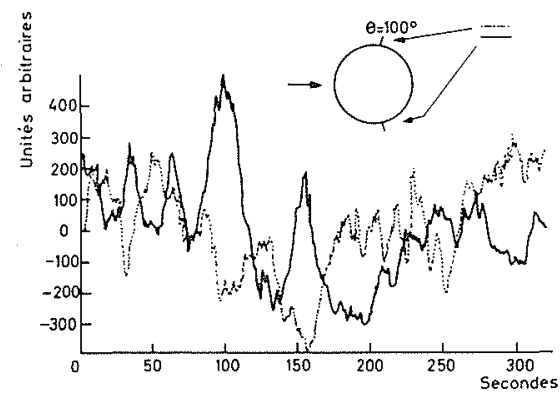

Comparaison des fluctuations de pression au col dans les 2 zones de séporation (BUGEY)

Figure 12
Cet échappement alterné est mis en évidence :

- sur les spectres de pression, par l'apparition d'un pic relativement étalé, centré sur une fréquence adimensionnelle de 0,18 et par l'apparition de hautes fréquences, juste après la séparation (fig. 13);

- sur la distribution azimutale des corrélations construites avec un capteur dans cette zone (fig. 14), qui fait apparaître une forte anti-corrélation entre les deux zones de séparation. Cette opposition de phase est par ailleurs confirmée par l'analyse interspectrale des fluctuations dans ces deux zones et par les visualisations des pressions (fig. 13).

Cet échappement tourbillonnaire est difficile à étudier à partir d'enregistrements sur site, car il correspond à des fréquences de l'ordre de 2 à $6.10^{-2}$ Hertz. Il est d'autre part contrarié par la turbulence atmosphérique et par le faible allongement de l'obstacle.

\section{Fluctuations de pression dans le sillage (Zone C)}

Dans le sillage, les fluctuations de pression sont faibles et les écarts-types constants en azimut. Il semble cependant que les parties hautes et basses de la tour soient le siège de fluctuations de haut niveau.

\section{Réfrigérant en interaction}

Un réfrigérant en interaction est soumis à un écoulement très perturbé qui possède en général un haut niveau de fluctuations.

On observe en conséquence, par rapport à la configuration isolée, une augmentation considérable des écartsteur de la tour (fig. 12).

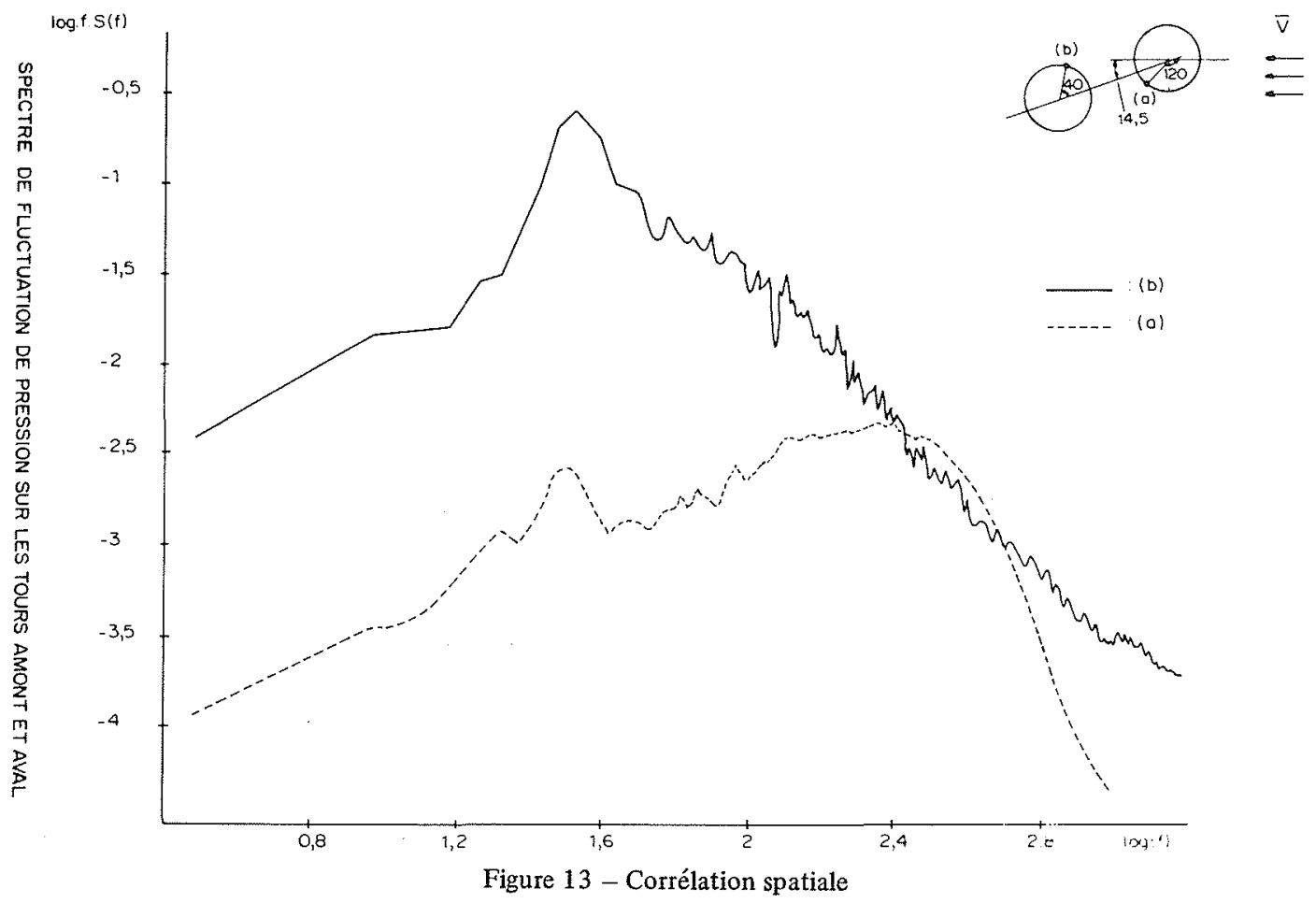




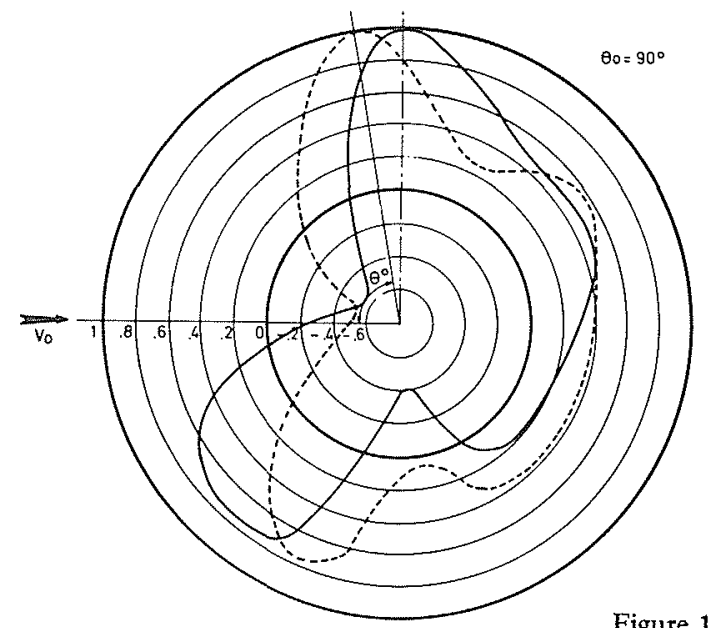

Figure 14 - Corrélation spatiale

types de pression qui peuvent être multipliés par 3 ou 4 en cas d'interaction forte (fig. 9) et une modification des distributions azimutales d'écarts-types. Les maximums de fluctuations apparaissent à l'endroit où les extrémités du sillage de la tour amont viennent rencontrer la coque (fig. 8).

L'analyse spectrale de ces fluctuations, sur la partie amont de la tour où se produisent les maximums, confirme l'augmentation considérable des fluctuations par rapport à la configuration isolée (fig. 8 et 13) et l'identité des fréquences des fluctuations sur les tours amont et aval.

Dans la partie arrière, l'échappement tourbillonnaire est en partie détruit et l'écart-type de la pression reste constant en azimut.

\section{Références}

[1]SAGEAU J.F. - Etude in situ du champ de pression stationnaire et instationnaire autour d'une cheminée de $240 \mathrm{~m}$, Journal of Industrial arodynamics 2 (1977/1978) p. 361-383.

[2] SAGEAU J.F. - L'action du vent sur les grands aéroréfrigérants - Etude expérimentale du champ de pression à la surface des aéroréfrigérants, Symposium international sur les réfrigérants de grande hauteur en béton armé : I.A.S.S. (S-10/11/78).

[3] SCANLAN R.M., and SOLLENBERGER N.J. - "Pressure difference across the shell of a Hyperbolic natural draft cooling tower". Proceedings, Fourth international Conference on wind effects on buildings and structures - London, England, 1975.

[4] NIEMANN M.J. - "Ein windlastkonzept für naturzugkühltürme" Konstruktiver Ingenieurbau - berichte - Heft. 29/30, Vulkan-Verlag Essen - 1977

\section{Discussion}

M. le Président. - Merci M. SAGEAU de cet exposé très clair. Est-ce qu'il y a des questions?

M. MILAN. - Vous avez parlé de rugosité d'obstacles lisses et d'obstacles rugueux. Quel est l'ordre de grandeur de la rugosité par rapport au diamètre?

M. SAGEAU. - Sur site, le rapport entre la hauteur de rugosité au diamètre varie entre $10^{-6}$ et $10^{-4}$, la rugosité étant représentée par les imperfections de la construction dans le cas d'un réfrigérant lisse et par des nervures si le réfrigérant est rugueux.

En soufflerie, le rapport est de l'ordre de $10^{-2}$ soit $10^{2}$ à $10^{3}$ fois plus élevé.

M. SAGNER. - Vous avez montré une courbe de répartition de la pression au point d'arrêt qui est assez bizarre comparée à la courbe de la pression dynamique. Est-ce que vous avez une idée du champ de vitesse qui correspond?
M. SAGEAU. - Ces résultats assez récents ont été obtenus "in situ" à la suite d'un enregistrement où une génératrice de capteurs a eu la chance de se trouver sur la ligne d'arrêt. Si ils sont surprenants lorsqu'on se réfère à des obstacles de types cylindriques, ils peuvent cependant s'expliquer par la forte tridimensionnalité de la géométrie de l'obstacle qui engendre des écoulements ascendants et descendants que l'on a d'ailleurs pu visualiser en soufflerie.

M. SAGNER, - Quand vous parlez de valeur moyenne sur site, il s'agit de moyenne à quelle échelle ?

M. SAGEAU. - Tous les traitements sont faits à partir d'enregistrements où la vitesse du vent est stationnaire et la direction constante. Dans ces conditions, les valeurs moyennes calculées sur 1,3 ou $10 \mathrm{mn}$ sont identiques. Au-delà de $10 \mathrm{mn}$, les hypothèses de stationnarité et de direction constante ne sont plus vérifiées "in situ". 